\title{
MicroRNA-135a Inhibits Nasopharyngeal Carcinoma Cell Proliferation Through Targeting Interleukin-17
}

\author{
Li-Xin Wang ${ }^{\mathrm{a}, \mathrm{d}}$ Zhao-Peng Kang ${ }^{\mathrm{b}}$ Zhi-Chao Yang ${ }^{\mathrm{c}}$ Rui-Xia Mac Yan Tan ${ }^{\mathrm{b}}$ \\ Xian-Bing Peng ${ }^{d}$ Run-Zhi Daid Jin Lid Yang Yuc Min Xu ${ }^{a}$
}

aDepartment of Otorhinolaryngology, Head and Neck Surgery, the Second Affiliated Hospital of Xi'an Jiao Tong University, Xi'an, 'Department of Andrology, Renmin Hospital, Hubei University of Medicine, Shiyan, 'Department of Otorhinolaryngology, Head and Neck Surgery, General Hospital of Ningxia Medical University, Yinchuan, dDepartment of Otorhinolaryngology, Head and Neck Surgery, Renmin Hospital, Hubei University of Medicine, Shiyan, China

\section{Key Words}

MiR-135a • IL-17 • Proinflammatory cytokines $・$ Nasopharyngeal carcinoma

\begin{abstract}
Background/Aims: The objective of this study was to investigate the potential role of IL-17 in the development of nasopharyngeal carcinoma (NPC) and to screen microRNAs (miRNAs) that potentially target IL-17 in NPC cells. Methods: Blood was collected from NPC patients and normal subjects, and plasma IL-17 concentration was quantified by enzyme-linked immunosorbent assay. An immortalized normal human nasopharyngeal epithelial cell line, NP69, was treated with or without human IL-17 (15 ng/mL) for various times, and expression of IL-1B, IL-6, IL-12, and TNF- $\alpha$ mRNA was assessed by real-time reverse transcription PCR. The candidate miRNAs that potentially target IL-17 were predicted by a bioinformatics strategy. The selected miR-135a mimic was transfected into primary NPC cells, and cell proliferation was assessed by MTT assay. Results: The concentration of plasma IL-17 was significantly higher in the NPC patients $(92.5 \pm 7.3 \mathrm{pg} / \mathrm{mL})$ than in the control subjects $(56.8 \pm 2.9 \mathrm{pg} / \mathrm{mL})$. In response to IL-17 treatment, the mRNA expression of IL-1B and IL- 6 was significantly upregulated and reached a peak at $12 \mathrm{~h}$, followed by a slight decrease at $24 \mathrm{~h}$, while the mRNA expression of IL-12 and TNF- $\alpha$ was significantly upregulated at $12 \mathrm{~h}$ and remained high even at $48 \mathrm{~h}$ after exposure to IL-17. Moreover, miR-135a specifically targets IL-17 and was dramatically downregulated in NPC cells compared with NP69 cells. Transfection of exogenous miR-135a mimic resulted in significant suppression of IL-17 secretion and subsequent inhibition of NPC cell proliferation. Conclusions: Blood IL-17 was significantly higher in NPC patients compared with normal subjects. Expression of miR-135a in the cancer cells isolated from nasopharyngeal tumors was significantly lower than that in NP69 cells, and suppression of IL-17 by miR-135a mimic resulted in significant inhibition of NPC cell proliferation. These findings suggested that downregulation of miR-135a may contribute to the development of NPC via the mechanism of IL-17 stimulation of proinflammatory cytokine expression.




\section{Introduction}

Nasopharyngeal carcinoma (NPC) with high malignancy is one of the common malignant tumors in Southern China and Southeast Asia. The 5-year survival rate of NPC is less than $60 \%$ [1]. Studies have shown that Epstein-Barr virus infection, chemical carcinogens, and genetic susceptibility may be implicated in pathogenesis of NPC [2]. Histopathological examination revealed massive infiltration of inflammatory cells in the microenvironment of NPC, which is believed to play a role in NPC cell proliferation [3].

NPC was initially regarded as lymphoepithelioma, and abnormal immunity is considered to play an important role in the development of NPC [4]. In this regard, IL-17, secreted by $\mathrm{CD}^{+} \mathrm{T}$ cells, has been reported to promote tumor progression by anti-apoptotic and proangiogenic mechanisms [5, 6]. IL-17 expression is elevated in various kinds of human tumors such as cervical cancer, hepatocellular carcinoma, ovarian cancer, esophageal cancer, breast cancer, gastric cancer, and NPC [7-10]. IL-17 was proposed to exert significant effects on tumor initiation and progression by affecting the antitumor immune response, $[11,12]$ and thus a novel immune-evasion mechanism utilized by the tumors through the inhibition of T-helper 17 (Th17) cell development was proposed [13]. The role of IL-17 in the development of NPC, however, remains to be investigated.

MicroRNAs (miRNAs) are highly conserved, endogenous small noncoding RNAs (17-25 nucleotides in length) that repress gene expression by binding to the $3^{\prime}$ untranslated region ( $3^{\prime}$ UTR) of their target mRNAs, inducing their degradation or translational repression. Studies have indicated that miRNAs could be used as novel diagnostic and prognostic biomarkers, or therapeutic tools for cancer treatment [14-16]. However, the function of miRNAs in the regulation of cytokines in NPC patients remains largely undefined.

In this study, we found that the level of plasma IL-17 was significantly higher in NPC patients. Furthermore, IL-17 stimulated the expression of IL-1ß, IL-6, IL-12, and TNF- $\alpha$ in NPC cells. Importantly, we identified IL-17 as a direct target of miR-135a and transfection of exogenous miR-135a mimic into NPC cells resulted in significant suppression of IL-17 and subsequent inhibition of NPC cell proliferation.

\section{Materials and Methods}

\section{Patient samples}

A total of 17 NPC patients ( 9 men and 8 women) and 7 normal healthy subjects ( 5 men and 2 women) were enrolled in this study. Tumor tissues were collected from patients with NPC who were treated in the Department of Otolaryngology, Head and Neck Surgery at the Second Affiliated Hospital of Xi'an Jiao Tong University. Written informed consent was obtained from each participant. The study protocol was approved by The Ethical Committee of the Second Affiliated Hospital of Xi'an Jiao Tong University Hospital.

Cell culture, cell proliferation, and cytotoxicity assay

The immortalized normal human nasopharyngeal epithelial cell line NP69 was obtained from the Chinese Academy of Science (Beijing, China). The cells were cultured in Dulbecco's modified Eagle medium (DMEM, Gibco) supplemented with 10\% fetal bovine serum (FBS, Gibco) and grown in a humidified atmosphere of $5 \% \mathrm{CO}_{2}$ and $95 \%$ air.

Primary NPC cells were isolated from six randomly selected tumor tissues, which were removed by surgical resection. Briefly, tumor tissues were washed with sterile phosphate buffered saline (Gibco). The tissues were then cut into $1-\mathrm{cm}^{3}$ pieces and grinded followed by digestion with $0.25 \%$ trypsin/ethylene diamine tetraacetic acid and $0.2 \%$ collagenase (type II, Clostridium histolyticum) overnight at $4{ }^{\circ} \mathrm{C}$. Digested tissues were resuspended in DMEM and filtered through a $55-\mu \mathrm{m}$ pore size nylon mesh. Filtered cells were counted and then transferred into 6-well plates in DMEM with 10\% FBS.

Cell proliferation and cytotoxicity assays were performed using colorimetric 3- [4, 5-dimethylthiazol2-yl]. -2, 5-diphenyltetrazolium bromide (MTT) following the manufacturer's instructions (Sigma Aldrich).

\section{KARGER}


Analysis of IL-17 production

Blood was harvested from NPC patients and control subjects, and plasma was isolated by centrifugation. Plasma IL-17 concentration was quantified using an enzyme-linked immunosorbent assay (ELISA) kit (R\&D Systems) following the manufacturer's instructions and using the Luminex 100 detection system (Life Technologies, Paisley, UK).

\subsection{RNA extraction and quantitative real-time reverse transcription PCR (RT-PCR)}

Total RNA was extracted from the cultured cells using TRIzol reagent (Invitrogen) following the manufacturer's instructions. RT-PCR analysis was performed using the SuperScript III FirstStrand kit (Invitrogen) and TaqMan Gene Expression Assay (Thermo Fisher Scientific). The primer sequences used for amplification were as follows: IL-1ß, sense: CATTGAGCCTCATGCTCTGTT, anti-sense: CGCTGTCTGAGCGGATGAA; IL-6, sense: ACTCACCTCTTCAGAACGAATTG and antisense: CCATCTTTGGAAGGTTCAGGTTG; IL-12, sense: ACCCTGACCATCCAAGTCAAA, antisense: TTGGCCTCGCATCTTAGAAAG; TNF- $\alpha$, sense: CCTCTCTCTAATCAGCCCTCTG, antisense: GAGGACCTGGGAGTAGATGAG; glyceraldehyde 3-phosphate dehydrogenase (GAPDH), sense: TGTGGGCATCAATGGATTTGG, antisense: ACACCATGTATTCCGGGTCAAT. Experiments were performed with an ABI 7500HT System instrument (Bio-Rad Laboratories) using the following PCR protocol: one cycle at $95^{\circ} \mathrm{C}$ for $10 \mathrm{~min}$, followed by 40 cycles at $95^{\circ} \mathrm{C}$ for $15 \mathrm{~s}$ and $60^{\circ} \mathrm{C}$ for $1 \mathrm{~min}$ with optical readings at $60^{\circ} \mathrm{C}$. Gene expression was normalized to internal controls and fold changes were calculated using relative quantification $\left(2^{-\Delta \Delta C t}\right)$. U6 small nuclear RNA (U6) and GAPDH were used as endogenous controls.

\section{Luciferase reporter assay}

The mutant (MUT) or wild-type (WT) 3'UTR of IL-17 was subcloned into the pGL3 Luciferase Reporter Vector (Promega). In IL-17 MUT, the 7-bp sequence complementary to the $5^{\prime}$ sequence of miR-135a was deleted. Cells were cotransfected with miR-135a mimic, inhibitor, or scrambled control and the vectors carrying IL-17 MUT or WT 3'UTR using Lipofectamine 2000 (Invitrogen). Activities of firefly and Renilla luciferases were measured using a dual-luciferase assay system (Promega).

\section{Statistical analysis}

Data are expressed as the mean \pm SD and were analyzed with a two-tailed Student's $t$ test using GraphPad Prism 5 (GraphPad Software). $P<0.05$ was considered statistically significant.

\section{Results}

Comparison of IL-17 in NPC patients and normal subjects

We first evaluated plasma IL-17 levels between NPC patients and control subjects. The IL-17 concentration was significantly higher in the NPC patients $(92.5 \pm 7.3 \mathrm{pg} / \mathrm{mL})$ compared with control subjects (56.8 $\pm 2.9 \mathrm{pg} / \mathrm{mL}$, Fig. 1). Clinical data analysis suggested that a high IL-17 plasma level in NPC was correlated with pathologic stage of the tumor $(P<$ $0.05)$, but not with age or gender $(P>0.05)$ (data not shown).

Effect of IL-17 on cytokine mRNA expression in NP69 cells

Next, the effect of IL-17 on mRNA expression of IL-1ß, IL-6, IL-12, and TNF- $\alpha$ by NP69 cells was assessed in vitro in cell culture. As shown in Fig. 2, mRNA expression of IL-1ß and IL-6 was significantly higher and reached a peak at $12 \mathrm{~h}$ following IL-17 treatment, and then slightly decreased at $24 \mathrm{~h}$,
Fig. 1. Comparison of IL-17 concentration in patients with NPC and normal subjects. Plasma was harvested from NPC patients (n =17) and normal subjects ( $\mathrm{n}=$ 7), and IL-17 concentration was quantified by ELISA as described in the methods. Vertical axis: IL17 concentration (pg/mL); horizontal axis: groups. ${ }^{*} \mathrm{P}<0.05$. Data presented are the average of 17 patients' samples or 7 normal control samples. 
Fig. 2. Effect of IL-17 on cytokine mRNA expression by NP69 cells. NPC cells were cultured in the serum free medium with or without IL-17 for varying hours as indicated. Cells were then harvested and used for quantification of IL-6 (Panel A), IL-1ß (Panel B), TNF- $\alpha$ (Panel C), and IL-12 (Panel D) mRNA by real-time RT-PCR as described in the methods. Data presented are an average of three separate experiments with the NP69 cells.

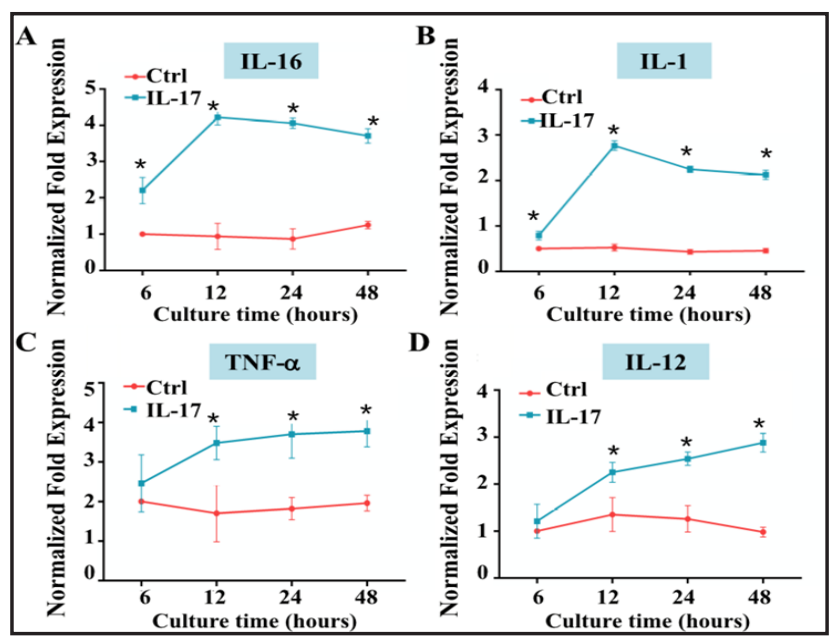

Fig. 3. Suppression of IL-17 by miR-135a. Panel A: Targeting sequence of IL-17 3'UTR by miR-135a. Panel B: Relative luciferase activity of IL-17 reporter. Firefly luciferase activity was assessed as described in the methods, and normalized to renilla luciferase activity and plotted as relative luciferase activity. Vertical axis: Relative luciferase activity; horizontal axis: wild type (WT) or mutant IL-17 3'UTR (Mut); solid bar: transfected with scramble miRNA; hatched bar: transfected with miR-135a. Data presented are one representative of three separate experiments.

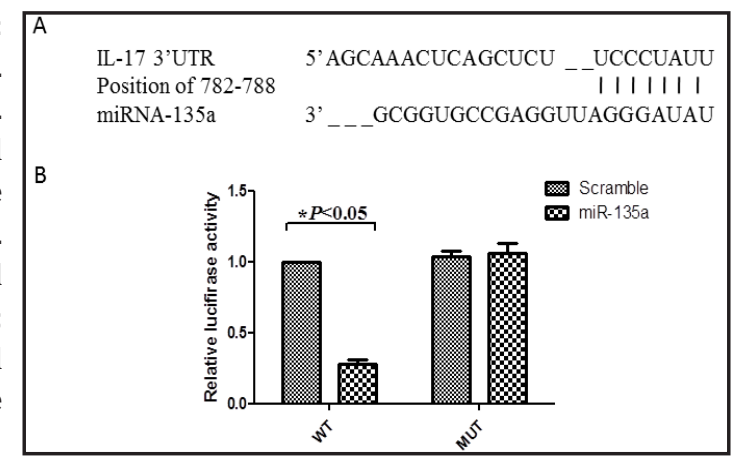

while mRNA expression of IL-12 and TNF- $\alpha$ was significantly higher at $12 \mathrm{~h}$ and remained high even at $48 \mathrm{~h}$ after exposure to IL-17.

\section{IL-17 was targeted by miR-135a}

Next, using TargetScan and PicTar, we predicted that miR-135a might directly target IL-17 (Fig. 3A). To experimentally test whether miR-135a targets IL-17, we constructed luciferase reporters by subcloning the WT or MUT 3'UTR of IL-17 downstream of the firefly luciferase cDNA in the pGL3 Luciferase Reporter Vector. We found that the relative luciferase activity of the reporter containing IL-17 WT 3'UTR was significantly decreased by approximately $70 \%$ upon miR-135a transfection as compared with the reporter containing IL-17 MUT 3'UTR in 293T cells (Fig. 3B). The cotransfection of miR-135a substantially decreased the luciferase activity of the WT reporter but barely affected that of the MUT reporter.

miR-135a was downregulated in primary NPC cells

Since miR-135a specifically targets IL-17, expression of miR-135a in primary NPC cells and normal (NP69) cells was compared by quantitative real-time PCR. As shown in Fig. 4, the expression of miR-135a was 72\% lower in primary NPC cells than in NP69 cells.

Next, following the transfection of miR-135a mimic or scrambled oligonucleotide into NPC cells, IL-17 concentration in the culture was quantified by ELISA and NPC cell proliferation was assessed by MTT assay. As expected, transfection of miR-135a mimic significantly upregulated the expression of miR-135a (Fig. 5A), which resulted in a more than $47 \%$ decrease in IL-17 synthesis compared with that in the cells transfected with scrambled oligonucleotide (Fig. 5B, P < 0.01). Furthermore, transfection of miR-135a mimic into primary NPC cells significantly inhibited proliferation from day 4 through day 8 (Fig. 5C, $P<0.01)$. 
Fig. 4. Comparison of miR-135a expression in the NPC cells and normal cells (NP69). Total RNA was extracted from the NPC cells and normal control cells (NP69). Expression of miR-135a in the NPC and normal cells were then quantified by real-time RT-PCR as described in the methods. Vertical axis: expression of miR-135a; horizontal axis: cells. ${ }^{*} \mathrm{P}<0.05$. Data presented are one representative experiment, which was performed three times using the NP69 cell line and one of the NPC cells.

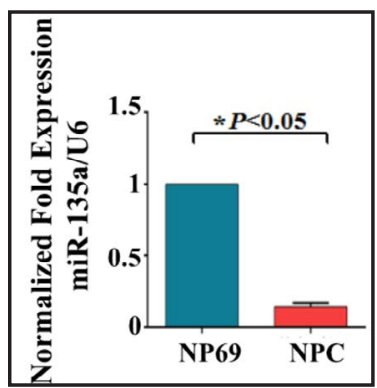

Fig. 5. Effect of exogenous miR-135a mimic on IL-17 synthesis and NPC cell proliferation. Panel A: Level of miR-135a following transfection of miR-135a mimic. Exogenous miR-135a mimic or scramble mimic were transfected into the NPC cells as described in the methods. Vertical axis: relative level of miR-135a over U6; horizontal axis: cells transfected with scramble mimic or miR-135a mimic (Mimic). Panel B: Effect of miR-135a mimic on IL-17 synthesis. NPC cells were transfected with miR-135a mimic or scramble mimic. IL-17 concentration was quantified by ELISA. Vertical axis: IL-17 concentration (pg/mL); horizontal axis: cells transfected with nothing (Blank), scramble mimic, or miR-135a mimic (Mimic). Panel C: Effect of miR-135a mimic on NPC cell proliferation. NPC cells were transfected with scramble mimic or miR-135a mimic and allowed to growth for 8

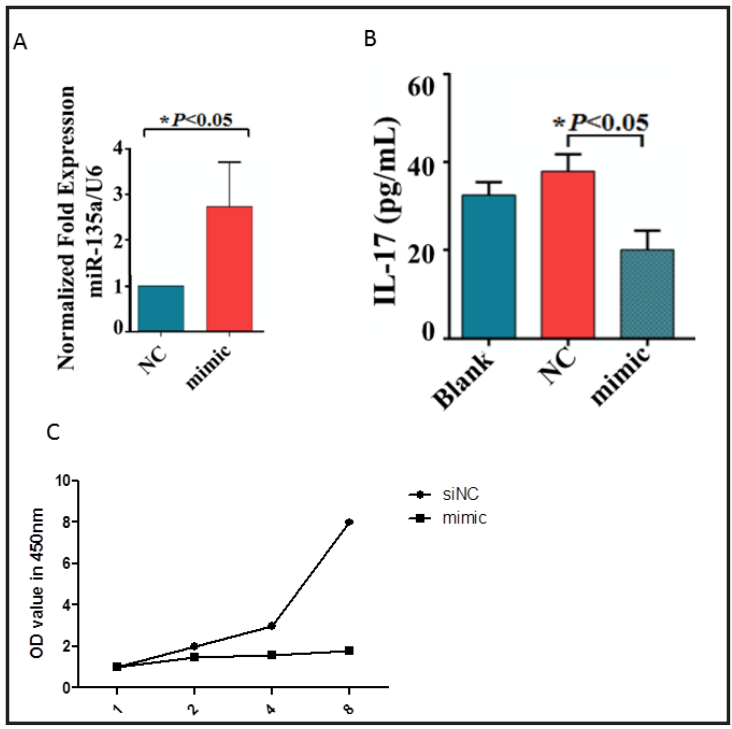
days. Cell number was assessed by MTT assay as described in the methods. Vertical axis: OD value at 450nm reading; horizontal axis: time (day); solid circle: cells transfected with scramble mimic; solid square: cells transfected with miR-135a mimic (mimic). Data presented are one representative of three separate experiments using three different NPC cells.

\section{Discussion}

IL-17 is an important proinflammatory cytokine secreted by Th17 cells. Despite the rapid expansion in studies investigating the role of IL-17 in the pathogenesis of autoimmunity and inflammatory diseases, its activities in cancer remain largely unknown. In the current study, we have investigated a potential role of IL-17 in the pathogenesis of NPC. We found that plasma IL-17 was significantly higher in patients with NPC compared to that in normal subjects, and that IL-17 significantly stimulated proinflammatory cytokines such as IL-6, IL$1 ß$, TNF- $\alpha$, and IL-12 in immortalized NP69 cells. In contrast, the level of miR-135a in tumor cells isolated from NPC tissues was significantly lower than that in the NP69 cell line. We further demonstrated that miR-135a targeted IL-17 and suppression of IL-17 by exogenous miR-135a mimic resulted in significant inhibition of NPC cell proliferation. These findings suggested that downregulation of miR-135a may lead to upregulation of IL-17, which contributes to the pathogenesis of NPC.

IL-17 has been reported to be involved in the process of immune regulation [17]. IL17 binds to its receptors to trigger downstream signaling cascades, which activates the production of inflammatory cytokines/chemokines and stimulates proliferation and differentiation of lymphocytes $[12,17]$. As a proinflammatory cytokine, IL-17 plays a role in host defense, immune surveillance, and regulation of tumor growth $[18,19]$. Elevation of IL17 expression has been demonstrated in a variety of malignancies, including ovarian cancer, hepatocellular carcinoma, breast cancer, prostate cancer, esophageal and gastric cancer, and non-melanoma skin cancers $[9,18,20,21]$. However, the role of IL-17 in NPC remains 
relatively unknown. In this regard, Li et al. have demonstrated that macrophage inhibitory factor can influence the survival of NPC through IL-17 [10]. Consistent with this observation, we demonstrated that the level of plasma IL-17 was significantly higher in NCP patients than in control subjects, suggesting IL-17 may be associated with NPC formation.

IL-17 could indirectly facilitate tumor progression through stimulating other cytokines/ chemokines [11]. For instance, IL-17 induces the release of the pro-angiogenic factor VEGF, which acts as a survival factor for tumor cells [22]. Furthermore, IL-17 promotes tumor growth through an IL-6/STAT3 signaling pathway [5]. However, the role of the IL-17stimulated cytokines in tumorigenesis remains controversial. In this regard, some animal studies revealed that IL-17 could protect animals from tumor outgrowth, while others revealed that IL-17 could promote tumor progression, depending on tumor origin and site [23]. The current study demonstrated that IL-17 stimulated IL-6, IL-1ß, TNF- $\alpha$, and IL-12 synthesis in NP69 cells, suggesting these cytokines may play a role in mediating the tumorpromoting effect of IL-17 in NPC.

miRNAs are known to regulate the expression of genes that induce mRNA decay or suppress translation. In the IL-17 signaling pathway, several miRNAs, including miR-23a, miRNA cluster 106a-363, miR-181a-5p, miR-15b, and miR-155, have been found to target signaling intermediates [24-27]. In this study, we further demonstrated that miR-135a expression was significantly lower in the NPC cells compared to that of normal cells, and found that miR-135a potentially targets IL-17, in that the 3'UTR of IL-17 mRNA contains the binding sequence for miR-135a. We have further demonstrated that ectopic expression of miR-135a in NPC cells significantly suppressed IL-17 synthesis, which resulted in significant inhibition of NPC cell proliferation. These findings suggested that downregulation of miR135a in NPC cells may result in upregulation of IL-17, and consequently, the higher level of IL-17 leads to proliferation of NPC cells. However, one limitation of this study was the relatively small case number of NPC subjects.

Taken together, the results of the current study demonstrated that miR-135a expression was significantly decreased in the NPC cells, while IL-17 secretion by NPC cells was significantly higher than that by normal cells. Furthermore, transfection of exogenous miR135a mimic into the NPC cells resulted in significant downregulation of IL-17 and inhibition of NPC cell proliferation. These findings suggested that targeting IL-17 through miR-135a could potentially be used as a therapeutic strategy for the treatment of NPC.

\section{Acknowledgements}

This study was supported by the Science and Technology Bureau Pilot Research Projects of Shiyan, Hubei Province (Grant No. 17Y26)

\section{Disclosure Statement}

The authors declare that there is no conflict of interests.

\section{References}

1 Genova P, Brunetti F, Bequignon E, Landi F, Lizzi V, Esposito F, Charpy C, Calderaro J, Azoulay D, de'Angelis $\mathrm{N}$ : Solitary splenic metastasis from nasopharyngeal carcinoma: A case report and systematic review of the literature. World J Surg Oncol 2016;14:184.

-2 Seto E, Yang L, Middeldorp J, Sheen TS, Chen JY, Fukayama M, Eizuru Y, Ooka T, Takada K: Epstein-barr virus (ebv)-encoded barf1 gene is expressed in nasopharyngeal carcinoma and ebv-associated gastric carcinoma tissues in the absence of lytic gene expression. J Med Virol 2005;76:82-88.

-3 Kneile JR, Tan G, Suster S, Wakely PE, Jr.: Expression of cd30 (ber-h2) in nasopharyngeal carcinoma, undifferentiated type and lymphoepithelioma-like carcinoma. A comparison study with anaplastic large cell lymphoma. Histopathology 2006;48:855-861. 


\section{Cellular Physiology Cell Physiol Biochem 2018;46:2232-2238 \begin{tabular}{l|l} 
DOI: 10.1159/000489591 & $\begin{array}{l}\text { O } 2018 \text { The Author(s). Published by S. Karger AG, Basel } \\
\text { www.karger.com/cpb }\end{array}$
\end{tabular}}

Wang et al.: MicroRNA-135a Inhibits NPC Cell Proliferation

4 Iezzoni JC, Gaffey MJ, Weiss LM: The role of epstein-barr virus in lymphoepithelioma-like carcinomas. Am J Clin Pathol 1995;103:308-315.

5 Wang L, Yi T, Kortylewski M, Pardoll DM, Zeng D, Yu H: Il-17 can promote tumor growth through an il-6stat3 signaling pathway. J Exp Med 2009;206:1457-1464.

6 Nam JS, Terabe M, Kang MJ, Chae H, Voong N, Yang YA, Laurence A, Michalowska A, Mamura M, Lonning S, Berzofsky JA, Wakefield LM: Transforming growth factor beta subverts the immune system into directly promoting tumor growth through interleukin-17. Cancer Res 2008;68:3915-3923.

7 Xu C, Yu L, Zhan P, Zhang Y: Elevated pleural effusion il-17 is a diagnostic marker and outcome predictor in lung cancer patients. Eur J Med Res 2014;19:23.

-8 Liu X, Jin H, Zhang G, Lin X, Chen C, Sun J, Zhang Y, Zhang Q, Yu J: Intratumor il-17-positive mast cells are the major source of the il-17 that is predictive of survival in gastric cancer patients. PLoS One 2014;9:e106834.

-9 Song Y, Yang JM: Role of interleukin (il)-17 and t-helper (th)17 cells in cancer. Biochem Biophys Res Commun 2017;493:1-8.

10 Li J, Mo HY, Xiong G, Zhang L, He J, Huang ZF, Liu ZW, Chen QY, Du ZM, Zheng LM, Qian CN, Zeng YX: Tumor microenvironment macrophage inhibitory factor directs the accumulation of interleukin-17-producing tumor-infiltrating lymphocytes and predicts favorable survival in nasopharyngeal carcinoma patients. J Biol Chem 2012;287:35484-35495.

11 Silva-Santos B, Serre K, Norell H: Gammadelta t cells in cancer. Nat Rev Immunol 2015;15:683-691.

12 Chen K, Kolls JK: Interluekin-17a (il17a). Gene 2017;614:8-14.

13 Kryczek I, Banerjee M, Cheng P, Vatan L, Szeliga W, Wei S, Huang E, Finlayson E, Simeone D, Welling TH, Chang A, Coukos G, Liu R, Zou W: Phenotype, distribution, generation, and functional and clinical relevance of th17 cells in the human tumor environments. Blood 2009;114:1141-1149.

14 Chen Y, Zhang J, Wang H, Zhao J, Xu C, Du Y, Luo X, Zheng F, Liu R, Zhang H, Ma D: Mirna-135a promotes breast cancer cell migration and invasion by targeting hoxa10. BMC Cancer 2012;12:111.

15 Lim LP, Lau NC, Garrett-Engele P, Grimson A, Schelter JM, Castle J, Bartel DP, Linsley PS, Johnson JM: Microarray analysis shows that some micrornas downregulate large numbers of target mrnas. Nature 2005;433:769-773.

16 Zhou Y, Li S, Li J, Wang D, Li Q: Effect of microrna-135a on cell proliferation, migration, invasion, apoptosis and tumor angiogenesis through the igf-1/pi3k/akt signaling pathway in non-small cell lung cancer. Cellular physiology and biochemistry : international journal of experimental cellular physiology, biochemistry, and pharmacology 2017;42:1431-1446.

17 Veldhoen M: Interleukin 17 is a chief orchestrator of immunity. Nat Immunol 2017;18:612-621.

18 Eyerich K, Dimartino V, Cavani A: Il-17 and il-22 in immunity: Driving protection and pathology. Eur J Immunol 2017;47:607-614.

19 Amatya N, Garg AV, Gaffen SL: Il-17 signaling: The yin and the yang. Trends Immunol 2017;38:310-322.

20 Nardinocchi L, Sonego G, Passarelli F, Avitabile S, Scarponi C, Failla CM, Simoni S, Albanesi C, Cavani A: Interleukin-17 and interleukin-22 promote tumor progression in human nonmelanoma skin cancer. Eur J Immunol 2015;45:922-931.

21 Alinejad V, Dolati S, Motallebnezhad M, Yousefi M: The role of il17b-il17rb signaling pathway in breast cancer. Biomed Pharmacother 2017;88:795-803.

22 Yang B, Kang H, Fung A, Zhao H, Wang T, Ma D: The role of interleukin 17 in tumour proliferation, angiogenesis, and metastasis. Mediators Inflamm 2014;2014:623759.

-23 Wilke CM, Kryczek I, Wei S, Zhao E, Wu K, Wang G, Zou W: Th17 cells in cancer: Help or hindrance? Carcinogenesis 2011;32:643-649.

24 Liu R, Ma X, Chen L, Yang Y, Zeng Y, Gao J, Jiang W, Zhang F, Li D, Han B, Han R, Qiu R, Huang W, Wang Y, Hao $\mathrm{J}$ : Microrna-15b suppresses th17 differentiation and is associated with pathogenesis of multiple sclerosis by targeting o-glcnac transferase. J Immunol 2017;198:2626-2639.

25 Kastle M, Bartel S, Geillinger-Kastle K, Irmler M, Beckers J, Ryffel B, Eickelberg O, Krauss-Etschmann S: Microrna cluster 106a 363 is involved in thelper 17 cell differentiation. Immunology 2017;152:402-413.

26 Cao Y, Zhao D, Li P, Wang L, Qiao B, Qin X, Li L, Wang Y: Microrna-181a-5p impedes il-17-induced nonsmall cell lung cancer proliferation and migration through targeting vcam-1. Cell Physiol Biochem 2017;42:346-356.

27 Na SY, Park MJ, Park S, Lee ES: Microrna-155 regulates the th17 immune response by targeting ets-1 in behcet's disease. Clin Exp Rheumatol 2016;34:S56-S63. 